\title{
ON THE SINGULARITIES OF LINEAR PARTIAL DIFFERENTIAL EQUATIONS*
}

\author{
BY \\ BERNARD OSGOOD KOOPMAN
}

The equations considered in this paper are linear systems of as many partial differential equations as there are unknown functions, of arbitrary order, and of coefficients analytic in the independent variables $\left(x_{1}, \cdots, x_{n}\right)$. We shall prove certain theorems regarding the region of existence of analytic solutions and the relation between the mobile singularities and the characteristics of the system. Our theorems have been proved in the case of one equation in two independent variables by Le Roux (Annales Scientifiques de l'École Normale Supérieure, (3), vol. 12 (1895), p. 227; Journal de Mathématiques, (5), vol. 4 (1898), p. 402), and in the real region under the same restrictions by Delassus (Annales Scientifiques de l'École Normale Supérieure, loc. cit., Supplement p. 53). They have been extended to equations in $n$ independent variables by these authors, and by Hadamard (Lectures on Cauchy's Problem, Yale University Press, 1923, p. 72), but only under the severest restrictions, and by methods which do not admit of generalization.

We shall consider the system

$$
L_{i}\left(u_{1}, \cdots, u_{m}\right)=0 \quad(i=1, \cdots, m),
$$

linear in the dependent variables $u$ and their partial derivatives with respect to $x_{1}, \cdots, x_{n}$ up to the order $r$. The fact that $r$ is the common highest order of the derivatives of all the $u$ 's in all the equations involves no restriction, for it may be brought about by means of suitable linear substitutions of the $u$ 's and the $L$ 's. The coefficients in (1) shall be analytic in $(x)=\left(x_{1}, \cdots, x_{n}\right)$ at the point $(x)=\left(x^{0}\right)$.

Let us pass briefly in review the classical treatment of the Cauchy problem for the system (1).

The object is to find $m$ functions

$$
u_{i}=f_{i}\left(x_{1}, \cdots, x_{n}\right) \quad(i=1, \cdots, m),
$$

analytic at the point $(x)=\left(x^{0}\right)$, which, on substitution into (1), reduce it to an identity, and such that, on the preassigned analytic manifold through $\left(x^{0}\right)$ :

$$
S\left(x_{1}, \cdots, x_{n}\right)=0, S\left(x^{0}\right)=0, \sum_{j=1}^{n}\left|\frac{\partial S}{\partial x_{j}}\right|_{\left(x_{0}\right)} \neq 0,
$$

* Presented to the Society, October 27, 1928; received by the editors in February, 1929. 
the functions (2) and their first $r-1$ derivatives take on the preassigned analytic values

$$
\begin{aligned}
& \frac{\partial^{\lambda} u_{i}}{\partial x_{1}^{\lambda_{1}} \cdots \partial x_{n}^{\lambda_{n}}}=\phi_{\lambda_{1} \cdots \lambda_{n}}^{i}\left(x_{1}, \cdots, x_{n}\right), \\
& \left(0 \leqq \lambda=\lambda_{1}+\cdots+\lambda_{n} \leqq r-1 ; \lambda_{i}=0,1, \cdots, r-1\right) .
\end{aligned}
$$

Here the 0th derivative means the function undifferentiated. The $\phi$ 's must, of course, satisfy certain conditions of compatibility; thus, for example, we must have for all $(x)$ on (3):

$$
d \phi_{0}^{i} \ldots 0=\phi_{1}^{i} \ldots 0 d x_{1}+\cdots+\phi_{0 \ldots 1}^{i} d x_{n} .
$$

By hypothesis one of the derivatives of $S$-let us say $\partial S / \partial x_{n}-$ is not zero at $\left(x^{0}\right)$. The transformation

$$
\begin{aligned}
& x_{1}^{\prime}=x_{1}, \cdots, x_{n-1}^{\prime}=x_{n-1}, \\
& x_{n}^{\prime}=S\left(x_{1}, \cdots, x_{n}\right)
\end{aligned}
$$

is, then, analytic and of analytic inverse near $\left(x^{0}\right)$. It transforms (1) and (4) into equations of the same form, but with (3) replaced by

$$
x_{n}^{\prime}=0 \text {. }
$$

The new conditions of compatibility, (5) etc., will not contain $x_{n}$ or $d x_{n}^{\prime}$, and indeed (4) and (5) etc., may be replaced by the following simpler initial data, in which the $\phi_{\lambda}{ }^{\prime}{ }^{\prime}$ 's are freed from all relations of the type (5):

$$
\begin{aligned}
\left(\frac{\partial^{\lambda} u_{i}}{\partial x_{n}^{\prime \lambda}}\right)_{x_{n^{\prime}=0}}= & \phi_{\lambda}^{\prime i}\left(x_{1}^{\prime}, \cdots, x_{n-1}^{\prime}\right) ; \\
& (\lambda=0,1, \cdots, r-1 ; i=1, \cdots, m) .
\end{aligned}
$$

The new equations obtained from (1) by the substitution (6) constitute $m$ linear equations in the $m$ quantities $\partial^{r} u_{i} / \partial x_{n}{ }^{\prime r}$ for which they may be solved, at least formally, so that they yield

$$
\frac{\partial^{r} u_{i}}{\partial x_{n}^{\prime}{ }^{\prime}}=l_{i} \quad(i=1, \cdots, m) .
$$

The $l$ 's are linear polynomials in the $u$ 's and their derivatives other than those appearing on the left; each of their coefficients is the quotient of a polynomial in the coefficients of the $L$ 's and in $S$ and its derivatives, divided by a certain determinant $D$; hence the equations (9) exist effectively, and their coefficients are analytic at $\left(x^{\prime 0}\right) \sim\left(x^{0}\right)$, provided $D \neq 0$ at this point.

The determinant $D$ is found on computation to be a homogeneous poly- 
nomial of degree $m r$ in the first derivatives of $S$, the coefficients being themselves homogeneous of degree $m r$ in the coefficients of the $L$ 's. The determinant $D$, regarded as a differential operator, shall be denoted by $D(S)$. In general, if the analytic manifold $F\left(x_{1}, \cdots, x_{n}\right)=0$ is such that

$$
\sum_{j}\left|\partial F / \partial x_{j}\right| \not \equiv 0 \text { and } D(F)_{F=0}=0
$$

this manifold is said to be a characteristic of the system (1). If the two preceding relations hold merely at $\left(x^{0}\right)$, the manifold is said to be tangent at this point to a characteristic.

We shall complete the solution under the supposit on that (3) is not tangent at $\left(x^{0}\right)$ to a characteristic, i.e., $D(F)_{\left(x^{0}\right)} \neq 0$, and that the equations of the problem are reduced to the form (9) and (8); and, further, that $\left(x^{\prime}{ }^{0}\right)=(0)$,-i.e., $x_{j}^{\prime}$ is replaced by $x_{j}^{\prime}-x_{j}{ }^{0}$.

By introducing certain derivatives of $u_{i}$ as further unknowns, our equations are replaced by an increased number of equations in an equal number of unknown functions, but involving only derivatives of the first order. Thus, after a change of notation, (9) and (8) are replaced by the equations

$$
\begin{gathered}
\frac{\partial u_{i}}{\partial x_{m}}=\sum_{\alpha=1}^{m} \sum_{\beta=1}^{n-1} A_{\alpha \beta}^{i} \frac{\partial u_{\alpha}}{\partial x_{\beta}}+\sum_{\alpha=1}^{m} B_{\alpha}^{i} u_{\alpha}+C^{i}(i=1, \cdots, m) ; \\
\left(u_{i}\right)_{x_{n}=0}=\phi_{i}\left(x_{1}, \cdots, x_{n-1}\right) .
\end{gathered}
$$

For the details of this reduction, cf., e.g., Goursat and Bourlet, Lecons sur l'Intégration des Equations aux Dérivées Partielles du Premier Ordre, Paris, Hermann, 1891, Chap. I.

We shall assume that in (10) $B \equiv C \equiv 0$. This entails no loss of generality; for consider the Cauchy problem with the differential equations

$$
\begin{array}{lrl}
\frac{\partial U_{i}}{\partial x_{n}}=\sum_{\alpha=1}^{m} \sum_{\beta=1}^{n-1} A_{\alpha \beta}^{i} \frac{\partial U_{\alpha}}{\partial x_{\beta}}+\sum_{\alpha=1}^{m} x_{0} B_{\alpha} i \frac{\partial U_{\alpha}}{\partial x_{0}}+x_{\alpha} C^{i} \frac{\partial U_{0}}{\partial x_{0}}, & \\
\frac{\partial U_{0}}{\partial x_{n}}=0 & (i=1, \cdots, m) ;
\end{array}
$$

and the following conditions for $x_{n}=0$ :

$$
U_{i}=x_{0} \phi_{i}\left(x_{1}, \cdots, x_{n-1}\right), \quad U_{0}=x_{0},
$$

$x_{0}$ being a new independent variable. This system is of the homogeneous form; and it is equivalent to (10) (11). For if $u_{i}$ is a solution of the latter, $U_{i}=x_{0} u_{i}, U_{0}=x_{0}$, is a solution of the former, and conversely. The first statement is proved by substitution; the second results from the computation, 
by means of the above equations, of the coefficients in the formal expansion of $U_{i}$ in powers of the $x$ 's.

We have, then, to consider the system

$$
\frac{\partial u_{i}}{\partial x_{n}}=\sum_{\alpha=1}^{m} \sum_{\beta=1}^{n-1} A_{\alpha \beta}^{i} \frac{\partial u_{\alpha}}{\partial x_{\beta}} \quad(i=1, \cdots, m),
$$

along with the Cauchy data (11). The problem is solved by the calcul des limites of Cauchy. First, it is shown that (11) and (12) suffice exactly to determine the formal expansions of the functions $u_{i}$ in power series in $x_{1}, \cdots, x_{n}$. Then it is proved that these formal series converge in the domain of convergence of solutions of a second system like (11) (12), in which $\phi_{i}$ and $A_{\alpha \beta}^{i}$ are replaced by power series whose coefficients are positive and greater than the absolute values of the corresponding coefficients in $\phi_{i}$ and $A_{\alpha \beta}^{i}$ of the given equations (11), (12) (cf. Goursat-Bourlet, loc. cit.).

It is at this point that we depart from the classical treatment, inasmuch as our special assumption of the linearity of the system (1) enables us to make use of dominating equations whose coefficients do not contain the unknown functions themselves (contrast: Goursat-Bourlet, loc. cit., p. 6: (2); our $A$ 's contain only $(x)$, while in this reference they are functions of $(u))$.

By hypothesis, the functions $A_{\alpha \beta}^{i}$ and $\phi_{i}$ are analytic in and on the boundary of a certain neighborhood of $(x)=(0)$ :

$$
\left|x_{j}\right| \leqq a \quad(j=1, \cdots, n) .
$$

Hence, for all (x) in (13), and for a suitable $M>0$ and $N>0$,

$$
\left|A_{\alpha \beta}^{i}\right|<M, \quad\left|\phi_{i}\right|<N .
$$

We shall, accordingly, make use of the following dominating equations:

$$
\begin{aligned}
\frac{\partial v_{i}}{\partial x_{n}} & =\frac{M \sum_{\alpha=1}^{m} \sum_{\beta=1}^{n-1} \frac{\partial v_{\alpha}}{\partial x_{\beta}}}{\left(1-\frac{x_{1}+\cdots+x_{n-1}}{a}\right)\left(1-\frac{x_{n}}{a}\right)}, \\
\left(v_{i}\right)_{x_{n}=0} & =\frac{N}{1-\frac{x_{1}+\cdots+x_{n-1}}{a}} .
\end{aligned}
$$

To solve these, we set $x_{1}=\cdots=x_{n-1}=y, x_{n}=z, v_{1}=\cdots=v_{m}=v$; the equations become

$$
\frac{\partial v}{\partial z}-\frac{M m(n-1) a^{2}}{[a-(n-1) y][a-z]} \frac{\partial v}{\partial y}=0,(v)_{z-0}=\frac{N a}{a-(n-1) y} .
$$


The first is reduced in the familiar manner to an ordinary differential equation, which is integrated by the separation of variables. The solution of the equations is found to be

$$
v=N a\left\{[a-(n-1) y]^{2}+2 M m(n-1)^{2} a^{2} \log \left(1-\frac{z}{a}\right)\right\}^{-1 / 2} ;
$$

- more precisely, that branch of the radical which reduces to $\mathrm{Na} /[\mathrm{a}-$ $(n-1) y]$ when $z=0$. The formal solution of (12) is analytic in a neighborhood $|y|<b,|z|<b(b \leqq a)$, determined by the conditions that (16) shall be analytic within it. But the radicand in (16) depends only on $M$ and $a$ and not on $N$. Hence (14) admits a solution analytic in $\left|x_{i}\right|<b, b$ independent of $N$.

These results insure the existence of a solution of the Cauchy problem (11) (12), analytic in the neighborhood $\left|x_{j}\right|<b$, where $b$ depends on the initial data $\phi_{i}$ only insofar as $\phi_{i}$ must be known to converge for $\left|x_{j}\right|<a$. Furthermore, the substitutions by means of which the general Cauchy problem (1) (3) (4) was reduced to the form (11) (12) involve in no wise the functions $\phi_{\lambda_{1} \ldots \lambda_{n}}^{i}$. From whence follows

THEOREM I. If the coefficients of $L_{i}$ and the function $S$ are analytic in the neighborhood

$$
\left|x_{i}-x_{i}{ }^{0}\right|<\alpha \quad(j=1, \cdots, n),
$$

throughout which $D(S)>$ const. $>0$, there will exist a certain fixed neighborhood

$$
\left|x_{j}-x_{i}{ }^{0}\right|<\beta \quad(j=1, \cdots, n)
$$

in which the Cauchy problem for $L_{i}=0$ and $S=0$ has an analytic solution. In the problem in question, the Cauchy data on $S=0$ are arbitrary, subject merely to the restriction of being analytic at all points of (17) lying on $S=0$.

THEOREM II. If the coefficients of $L_{i}$ and the function $S$ are analytic at the point $(x)=(X)$, and if $D(S)_{(X)} \neq 0$, there will exist a closed neighborhood

$$
\left|x_{j}-X_{j}\right| \leqq \gamma \quad(j=1, \cdots, n),
$$

and a constant $\beta>0$, such that, if $\left(x^{0}\right)$ be taken arbitrarily in (19), and if analytic Cauchy data be assigned on that portion of the manifold $S(x)=S\left(x^{0}\right)$ which lies in the larger neighorhood

$$
\left|x_{j}-X\right|<2 \gamma \quad(j=1, \cdots, n),
$$

the corresponding Cauchy problem will admit a solution analytic in (18). It is to be emphasized that $\beta$ is independent both of $\left(x^{0}\right)$ and of the Cauchydata.

For the proof, we have but to take $\gamma$ so small that throughout (20) $L_{i}$ 
and $S$ are analytic, and $D(S)>$ const. $>0$. Then if $\left(x^{0}\right)$ is taken such that $\left|x_{j}{ }^{0}-X\right| \leqq \gamma$, and $\alpha=\gamma$, Theorem I will apply. Since this furnishes a positive $\beta$ for each point $\left(x^{0}\right)$ of the closed neighborhood (19), we have but to take for the $\beta$ of the present theorem their lower limit, which is positive.

When the process of analytic continuation is applied in an obvious manner, the following theorem is obtained:

THEOREM III. Let $R_{2 n}$ be a $2 n$-dimensional region of the complex $x_{1} \cdots x_{n-}$ space; let $L_{i}$ have analytic coefficients in $R_{2 n}$; finally, let $S_{2 n-2}$ be a $(2 n-2)$ dimensional region of the analytic manifold $S\left(x_{1}, \cdots, x_{n}\right)=0$, where $S_{2 n-2}$ lies in $R_{2 n}$, and at no point of $S_{2 n-2}$ does $S\left(x_{1}, \cdots, x_{n}\right)$ satisfy the equation of characteristics $D(S)=0$. There will then exist a $2 n$-dimensional subregion $P_{2 n}$ of $R_{2 n}$, containing $S_{2 n-2}$, and such that, whatever be the analytic data on $S_{2 n-2}$, the Cauchy problem will admit a solution analytic throughout $P_{2 n}$.

In this theorem the coefficients of $L_{i}$ as well as the Cauchy data may be multiple-valued.

THEOREM IV. Let the coefficients of $L_{i}$ be analytic in the $2 n$-dimensional region $R_{2 n}$. If $u_{1}, \cdots, u_{n}$ are solutions of $L_{i}=0$ which are analytic in $R_{2 n}$ except at all points of an analytic manifold $S_{2 n-2}$ lying in $R_{2 n}$, then $S_{2 n-2}$ is a characteristic manifold of $L_{i}=0$.

Suppose the theorem false. Then we could find a point $(X)$ on $S_{2 n-2}$ in a sufficiently small neighborhood of which $S_{2 n-2}$ is represented by the equation $S(x)=S(X)$, where $S(x)$ is analytic, and $D(S) \neq 0$, at $(X)$. Let Theorem II be applied to $L_{i}, S,(X)$, and let the point $\left(x^{0}\right)$ be so chosen that

$$
\left|x_{j}{ }^{0}-X\right|<\frac{\beta}{2} \quad(j=1, \cdots, n),
$$

and that $S\left(x^{0}\right) \neq S(X)$. Evidently the functions $u_{1}, \cdots, u_{n}$ are analytic at those points of the manifold $S(x)=S\left(x^{0}\right)$ which lie in (20), for it is an easy matter to insure that all our points be in $R_{2 n}$. Hence, by Theorem II, analytic solutions of $L_{i}=0$ exist in (18) which take on the values of $u_{1}, \cdots, u_{n}$ and of appropriately chosen ones of their derivatives on the manifold $S(x)=$ $S\left(x^{0}\right)$. By the uniqueness of the analytic solution of Cauchy's problem, these solutions are identical with the original solutions $u_{1}, \cdots, u_{n}$. But, by (21), the neighborhood of their analyticity (18) contains $(X)$. But this is contradictory to the hypothesis that $(X)$, which is on $S_{2 n-2}$, is a singularity of $u_{1}, \cdots, u_{m}$. The truth of the theorem follows.

It is to be emphasized that these results are not in general true when stated for the real domain. Thus, if Theorem I were true for Laplace's 
equation in real space, there could be no such thing as a solution with an isolated point singularity. As for Theorem IV in the real domain, even with equations with real characteristics, we may consider the equation

$$
\frac{\partial^{2} u}{\partial x^{2}}+\frac{\partial^{2} u}{\partial y^{2}}-\frac{\partial^{2} u}{\partial z^{2}}=0
$$

and the solution $u=f(x, y)$, where $f(x, y)$ satisfies

$$
\frac{\partial^{2} u}{\partial x^{2}}+\frac{\partial^{2} u}{\partial y^{2}}=0
$$

and admits the circle $x^{2}+y^{2}=1$ as a locus of essential singularities, without, however, being singular near this circle. This gives an example of a function satisfying the hypotheses of Theorem IV for reals, with $S_{2 n-2}$ as the cylinder $x^{2}+y^{2}=1$, which singularity is not a characteristic: if $S=x^{2}+y^{2}-1$,

$$
\left(\frac{\partial S}{\partial x}\right)^{2}+\left(\frac{\partial S}{\partial y}\right)^{2}-\left(\frac{\partial S}{\partial z}\right)^{2}=4 S+4
$$

Columbia UnIversity, New York, N. Y. 